\title{
Recent advances in the application of data science and machine/deep learning methods to research in chemical and biological sciences : a review
}

\author{
Ben Geoffrey A S \\ Independent Researcher \\ ORCID ID : https://orcid.org/0000-0001-6545-6314 \\ Corresponding Email : bengeof@gmail.com
}

\begin{abstract}
The rise in application of methods of data science and machine/deep learning in chemical and biological sciences must be discussed in the light of the fore-running disciplines of bio/cheminformatics and computational chemistry and biology which helped in the accumulation of enormous research data because of which successful application of data-driven approaches have been made possible now. Many of the tasks and goals of Ab initio methods in computational chemistry such as determination of optimized structure and other molecular properties of atoms, molecules, and compounds are being carried out with much lesser computational cost with datadriven machine/deep learning-based predictions. One observes a similar trend in computational biology, wherein, data-driven machine/deep learning methods are being proposed to predict the structure and dynamical of interactions of biological macromolecules such as proteins and DNA over computational expensive molecular dynamics based methods. In the cheminformatics space, one sees the rise of deep neural network-based methods that have scaled traditional structureproperty/structure-activity to handle big data to design new materials with desired property and drugs with required activity in deep learning-based de novo molecular design methods. In the bioinformatics space, data-driven machine/deep learning approaches to genomic and proteomic data have led to interesting applications in fields such as precision medicine, prognosis prediction, and more. Thus the success story of the application of data science, machine/deep learning, and artificial intelligence to the disciple of chem/bio-informatics, and computational chemistry and biology has been told in light of how these fore-running disciplines had created huge repositories of data for data-driven approaches to be successful in these disciplines.
\end{abstract}




\section{Introduction}

When one wishes to discuss the recent research advances made in the application of data science and machine/deep learning to chemical and biological sciences, one has to also discuss the forerunning disciplines of computational chemistry and biology and chem/bio-informatics where digital technology helped in advancing research in chemical and biological sciences. In fact, it is these fore-running disciplines over the past years have helped in the accumulation of enormous research data related to chemistry and biology which have made now a fertile ground to apply the datahungry disciplines of data science and machine/deep learning to advance research in chemistry and biology through the power of computers and digital technology. Therefore the course of the review would entail, first to broadly outline these fore-running computational and informatics disciplines, then to discuss some major data science and machine/deep learning algorithms and finally their application by different research groups in advancing research in chemical and biological sciences.

\section{Lighting introduction to some fore-runners}

\subsection{Computational chemistry and chem-informatics}

The dawn of computers and their computational power, saw the increased interest to harness the computational power of computers to solve research problems through numerical methods[1-6]. In Chemistry, the attempts to numerically solve the many-body quantum problem by numerical approximations to the Schrodinger equation led to many methods such as Hartree-Fock and Density Functional Theory methods. By solving the multi-electron system of the atom numerically, it was possible to know the structural, electronic, optoelectronic, thermodynamic, and other properties of atoms and compounds at quantum mechanical level theory[7-15]. With a large amount of chemical descriptor data availability, chem-informatic space saw the rise in statistical relations being derived between desired material properties and chemical descriptors in what came to be known as Quantitative Structure-Property/Structure-Activity studies and is said to have accelerated new material or drug molecule discovery [16-21].

Rdkit is a popular cheminformatics package for fetching chemical descriptors, molecular fingerprints of a given compound and to perform similar searches of a given compound [22] 


\subsection{Computational biology and bio-informatics}

In computational biology, the computational power of computers is used to model structure, behaviour, and interactions of biological macromolecules such as protein and DNA at classical level theory. Empirical based scoring functions and force fields have been used to study the interaction of protein-ligand, protein-protein, and protein-DNA through molecular docking methods. Newton's equations for many-body systems have been solved numerically to model the dynamic behaviours of biological macromolecular systems involving proteins, DNA and lipid membranes in molecular dynamic simulations to theoretically elucidate the structure and other physio-chemical properties of interest [23-36]. With high throughput sequencing capabilities dawned a new advent of a huge amount of genomic and proteomic data. [37]. A comparison of nucleotide and amino acid sequences can be performed using BLAST and other such algorithms in bio-informatics space to learn about the functional and evolutionary relationships of the compared sequences [38].

Bio-conductor is a popular bioinformatics package for fetching genomic and proteomic data and performing BLAST and other sequence similarity algorithms to help identify functional and evolutionary relationships of the compared sequences [39].

\subsection{Lighting introduction to methods in data science and machine/deep learning}

Data science is the art of uncovering hidden patterns in raw data by applying data science and machine learning algorithms. This is useful to generate scientific insights from raw data otherwise not understood [40-49]. One of the major chunks of the work of a data scientist is data preprocessing and feature engineering and prepare the data for the application of data science and machine learning algorithms [50,51]. Python is a programming language in which many standard libraries are present to perform these operations. Bio-python and bio-pandas are some standard python libraries for fetching bio-chem related data and prepare and clean up the data through the process of data wrangling before the application of data science and machine learning algorithms $[52,53]$. In the case of handling big data and larger than memory data wrangling tasks, the library Dask can be used [54]. In the process of feature engineering, transformers are used to scale, normalize data which improves prediction scores and to drop null values [55]. To reduce the dimensionality of data before applying the machine learning algorithms, dimensionality reduction methods such as Principal Component Analysis(PCA) are applied [56]. When one deals with data other than numerical data such as text data, the data should be vectorized for the machine learning algorithms to be able to deal with them [57]. To uncover hidden patterns from raw data some 
standard unsupervised machine learning algorithms used are clustering, k-means, and KNN (KNearest Neighbours). These are implemented using a standard python machine learning library scikit-learn [58]. To visualize and plot data, python's data visualization libraries such as seaborn, and matplotlib [59]. In cases of supervised machine learning where one is dealing with labelled data and where some input predictor variables are used to predict an output, a predicted variable, multivariate linear and non-linear regression algorithms are used for statistical correlation-based prediction and decision tree and random forests are used for probability decision tree-based prediction [60]. The data set is divided between training, validation, and prediction when applying these algorithms and hyper-parameter tuning is implemented using grid and randomized search to prevent over-fitting. To scale machine learning algorithms described above to handle big data, the library Py-Spark is used [61]. When there are intermediate layers of nodes with inter-connections between layers between the input and output node layer, such architectures are called deep learning models. Analogous to the biological neuron's firing based upon the neurons' activation potential principle, mathematical functions called activation functions are used to mathematically determine when and how the outputs of each layer are passed on as inputs to the next layer. Therefore together they bear the name deep neural networks. Tensorflow, PyTorch, and Keras are standard python libraries to implement deep neural networks of varied architectures. How these data science, machine/deep learning algorithms have been used on chemical and biological data by different research groups to solve different research problems are detailed in the next section [62].

\section{1}

\section{Machine/deep learning in the computational chemistry and chem-informatics space : a review}

For many years, Density Functional Theory (DFT) approximations to the Schrodinger equation has been standard first-principles method to solve the electronic structure problems of atoms, molecules, and compounds. The problem of finding the energy density functional for a particular system is solved via the Kohn-Sham equations. The energy density functional is a function of charge distribution in the system which in turn is a function in space and time and therefore in the solving system, the structure and dynamics are solved. Therefore finding the appropriate energy density functional is key to solving the DFT electronic structure problem. In a study published in 2012, John C Synder et al, showed how machine learning can be used in finding density functional [63]. In succeeding years, research groups have demonstrated how machine learning can be used to bypass the solving of the Kohn-Sham equations to solve the standard DFT problem of finding the energy functional of a system through machine learning-based methods [64-66]. 
In computational chemistry, one solves DFT system involving atoms, molecules, or compounds to know their structure, spectroscopic and other molecular properties. However in Machine/Deep Learning one finds alternate data-driven approaches to predicting structure [67] , spectra [68,69], and other molecular properties [70]. In the cheminformatics space, structure-property relationship studies were used to accelerate new material discovery. However, in the new age of Big data and AI, data driven machine learning based methods have taken over to accelerate new material discovery [71,72]. Choudhary, K. Et al demonstrate the use of data-driven machine learning-based method to accelerate the discovery of efficient solar cell materials [73], and similarly Masood, $\mathrm{H}$ et al demonstrate data-driven machine learning accelerated discovery of photocatalyst [74]. The JARVIS-DFT database of Quantum Chemical Descriptors for 3D and 2D materials is a recently published resource that is being utilized in data-driven new material discovery. Choudhary, K. Et al demonstrate the use of the JARVIS resource in the discovery of 2D and 3D thermoelectric materials [75].

Deep neural network architectures such as generative autoencoders, generative recurrent neural networks, generative adversarial neural networks, and graph convolution neural networks have been used to design new molecules for a required material property or in case of drug discovery. [76-80]

\section{2}

\section{Machine/deep learning in the computational biology and bio-informatics space : a review}

One observes a similar trend in computational biology and bioinformatics space wherein the computationally predictive tasks are now further buttressed by data-driven predictions in the age of Big data and AI through machine/deep learning. In computational biology, the knowledge of force fields for different chemical environments of the system of biological macromolecules is important to computationally solve the structure and dynamic interaction of biological macromolecules such as proteins and DNA. We find that researcher groups have reported force fields developed through machine learning and validated for atomistic and coarse-grained simulations [81-83]. Noé, F. Et al suggest that machine-learned force fields may soon replace conventionally used force fields in atomistic and coarse-grained simulations of proteins. Computational methods were earlier used to study protein-protein, protein-ligand, and protein-DNA interactions. In the age of Big Data and AI, we observe the data-driven approaches have taken over such tasks with much less computational cost. Sun, T et al report a deep learning tool that can be used for protein-protein interaction 
prediction, and Zeng, $\mathrm{M}$ et al report a deep learning-based tool for predicting the interaction site in case of protein-protein interaction [84,85]. Protein-ligand interaction predictions are significant to the field of small drug molecule discovery. Wang, Y. B. et al have developed a deep learning-based tool based on the LSTM neural network architecture to predict drug-target interaction or otherwise protein-ligand interaction [86]. Wen, M. Et al also report a deep learning-based tool for drug-target prediction [87]. Geoffrey AS et al have developed a hybrid approach for predicting drug-target interactions which combine both deep learning and computational-based approach [88]. Several research groups have developed deep learning-based tools to predict protein-ligand binding affinities [89-92]. Efforts to predict binding residues using deep learning have also been reported [93]. Traditionally, in the Bio-informatics space one deals with amino acid and nucleotide sequence and performs operations such as sequence comparison to elucidate protein function and evolutionary relationship. This data-rich discipline has become a fertile ground for Deep Learning methods to take over. Deep Learning-based tools have been developed to predict gene expression [94]. Differential gene expression data of normal and diseased persons have been used for example in the case of cancer, to classify the cancer subtype [95] and cancer prognosis prediction [96]. Along similar lines, Deep Learning based tools have been developed for disease risk prediction [97]. Also, machine learning-based methods have been used to deal with genomic data to identify biomarkers for diseases and to identify therapeutic targets [98,99]. While traditionally in bioinformatics one performs nucleotide sequence comparison with techniques such as BLAST to also understand evolutionary relationships, machine learning-based methods have also been developed to perform phylogenetic tree construction and classification and also taxonomic classification from DNA sequence [100-102]. In the proteomics space, deep learning tools have also been developed wherein given the amino acid sequence the deep learning tool predicts the protein structure and function [103, 104]

\section{Conclusion}

Thus the rise and the success story of the application of data science, machine/deep learning, and artificial intelligence to the disciple of chem and bio-informatics, and computational chemistry and biology has been told in the light of how these fore-running disciplines created huge repositories of data for data-driven approaches to be successful in these disciplines. 


\section{References}

1. Dykstra, C., Frenking, G., Kim, K., \& Scuseria, G. (Eds.). (2011). Theory and applications of computational chemistry: the first forty years. Elsevier.

2. Peyerimhoff, S. D. (2002). The development of computational chemistry in Germany. Reviews in computational chemistry, 18, 257-292.

3. McArdle, S., Endo, S., Aspuru-Guzik, A., Benjamin, S. C., \& Yuan, X. (2020). Quantum computational chemistry. Reviews of Modern Physics, 92(1), 015003.

4. Darden, L. (1997). Recent work in computational scientific discovery. In Proceedings of the Nineteenth Annual Conference of the Cognitive Science Society (pp. 161-166). Mahwah, New Jersey: Lawrence Erlbaum.

5. Boyd, R. J. (2000). The development of computational chemistry in Canada. Reviews in Computational Chemistry, 15, 213-299.

6. Willett, P. (2011). Chemoinformatics: a history. Wiley Interdisciplinary Reviews: Computational Molecular Science, 1(1), 46-56.

7. Parr, R. G. (1980). Density functional theory of atoms and molecules. In Horizons of quantum chemistry (pp. 5-15). Springer, Dordrecht.

8. Runge, E., \& Gross, E. K. (1984). Density-functional theory for time-dependent systems. Physical Review Letters, 52(12), 997.'

9. Wong, M. W. (1996). Vibrational frequency prediction using density functional theory. Chemical Physics Letters, 256(4-5), 391-399.

10. Koch, W., \& Holthausen, M. C. (2015). A chemist's guide to density functional theory. John Wiley \& Sons.

11. Dobson, J. F., Vignale, G., \& Das, M. P. (Eds.). (2013). Electronic density functional theory: recent progress and new directions. Springer Science \& Business Media.

12. Mackrodt, W. C., Harrison, N. M., Saunders, V. R., Allan, N. L., Towler, M. D., Apra, E., \& Dovesi, R. (1993). Ab initio Hartree-Fock calculations of $\mathrm{CaO}, \mathrm{VO}, \mathrm{MnO}$ and NiO. Philosophical Magazine A, 68(4), 653-666.

13. Habas, M. P., Dovesi, R., \& Lichanot, A. (1998). The phase transition in alkaline-earth oxides: a comparison of ab initio Hartree-Fock and density functional calculations. Journal of Physics: Condensed Matter, 10(31), 6897.

14. Karna, S. P., Prasad, P. N., \& Dupuis, M. (1991). Nonlinear optical properties of p-nitroaniline: An ab initio time-dependent coupled perturbed Hartree-Fock study. The Journal of chemical physics, 94(2), 1171-1181. 
15. Mansour, M., Issa, T. B., Issaoui, N., Harchani, A., Puebla, E. G., \& Ayed, B. (2019). Synthesis, crystal structure, vibrational spectroscopy, optical investigation and DFT study of a novel hybrid material: 4, 4'-diammoniumdiphenylsulfone iodobusmuthate. Journal of Molecular Structure, 1197, 478-486.

16. Colmenarejo, G., Alvarez-Pedraglio, A., \& Lavandera, J. L. (2001). Cheminformatic models to predict binding affinities to human serum albumin. Journal of medicinal chemistry, 44(25), 4370-4378.

17. Begam, B. F., \& Kumar, J. S. (2012). A study on cheminformatics and its applications on modern drug discovery. Procedia engineering, 38, 1264-1275.

18. Chen, M., Jabeen, F., Rasulev, B., Ossowski, M., \& Boudjouk, P. (2018). A computational structure-property relationship study of glass transition temperatures for a diverse set of polymers. Journal of Polymer Science Part B: Polymer Physics, 56(11), 877-885.

19. Martínez, M. J., Ponzoni, I., Díaz, M. F., Vazquez, G. E., \& Soto, A. J. (2015). Visual analytics in cheminformatics: user-supervised descriptor selection for QSAR methods. Journal of cheminformatics, 7(1), 39.

20. Buchwald, P., \& Bodor, N. (2002). Computer-aided drug design: the role of quantitative structure-property, structure-activity and structure-metabolism relationships (QSPR, QSAR, QSMR). Drugs Future, 27(6), 577-588.

21. Todeschini, R., Consonni, V., \& Gramatica, P. (2009). Chemometrics in QSAR.

22. Landrum, G. (2013). RDKit: A software suite for cheminformatics, computational chemistry, and predictive 8odelling.

23. Gauthier, J., Vincent, A. T., Charette, S. J., \& Derome, N. (2019). A brief history of bioinformatics. Briefings in bioinformatics, 20(6), 1981-1996.

24. Golding, G. B. (2003). DNA and the revolutions of molecular evolution, computational biology, and bioinformatics. Genome, 46(6), 930-935.

25. Nair, A. S. (2007). Computational biology \& bioinformatics: a gentle overview. Communications of the Computer Society of India, 2.

26. Ouzounis, C. A., \& Valencia, A. (2003). Early bioinformatics: the birth of a discipline-a personal view. Bioinformatics, 19(17), 2176-2190.

27. Hagen, J. B. (2000). The origins of bioinformatics. Nature Reviews Genetics, 1(3), 231-236.

28. Searls, D. B. (2010). The roots of bioinformatics. PloS Comput Biol, 6(6), e1000809.

29. Ragan, M. A., Littlejohn, T., \& Ross, B. (2008). Genome-scale computational biology and bioinformatics in Australia. PloS Comput Biol, 4(8), e1000068.

30. Brusic, V. (2007). The growth of bioinformatics. 
31. Wei, L., \& Yu, J. (2008). Bioinformatics in China: a personal perspective. PloS Comput Biol, 4(4), e1000020.

32. Mushegian, A. (2011). Grand challenges in bioinformatics and computational biology. Frontiers in genetics, 2, 60 .

33. Claverie, J. M. (2000). From bioinformatics to computational biology. Genome research, 10(9), 1277-1279.

34. Baxevanis, A. D., Bader, G. D., \& Wishart, D. S. (Eds.). (2020). Bioinformatics. John Wiley \& Sons.

35. Fenstermacher, D. (2005). Introduction to bioinformatics. Journal of the American Society for Information Science and Technology, 56(5), 440-446.

36. Aluru, S. (Ed.). (2005). Handbook of computational molecular biology. CRC Press.

37. Reuter, J. A., Spacek, D. V., \& Snyder, M. P. (2015). High-throughput sequencing technologies. Molecular cell, 58(4), 586-597.

38. Donkor, E. S., Dayie, N. T., \& Adiku, T. K. (2014). Bioinformatics with basic local alignment search tool (BLAST) and fast alignment (FASTA). Journal of Bioinformatics and Sequence Analysis, 6(1), 1-6.

39. Gentleman, R. C., Carey, V. J., Bates, D. M., Bolstad, B., Dettling, M., Dudoit, S., ... \& Hornik, K. (2004). Bioconductor: open software development for computational biology and bioinformatics. Genome biology, 5(10), R80.

40. Van Der Aalst, W. (2016). Data science in action. In Process mining (pp. 3-23). Springer, Berlin, Heidelberg.

41. Provost, F., \& Fawcett, T. (2013). Data science and its relationship to big data and datadriven decision making. Big data, 1(1), 51-59.

42. Dhar, V. (2013). Data science and prediction. Communications of the ACM, 56(12), 64-73.

43. Tansley, S., \& Tolle, K. (2009). The fourth paradigm: data-intensive scientific discovery (Vol. 1). T. Hey (Ed.). Redmond, WA: Microsoft research.

44. Hand, D. J., \& Adams, N. M. (2014). Data Mining. Wiley StatsRef: Statistics Reference Online, 1-7.

45. Flach, P. (2012). Machine learning: the art and science of algorithms that make sense of data. Cambridge University Press.

46. Xu, D., \& Tian, Y. (2015). A comprehensive survey of clustering algorithms. Annals of Data Science, 2(2), 165-193.

47. Singh, A., Thakur, N., \& Sharma, A. (2016, March). A review of supervised machine learning algorithms. In $20163^{\text {rd }}$ International Conference on Computing for Sustainable Global Development (INDIACom) (pp. 1310-1315). Ieee. 
48. Singh, A., Thakur, N., \& Sharma, A. (2016, March). A review of supervised machine learning algorithms. In $20163^{\text {rd }}$ International Conference on Computing for Sustainable Global Development (INDIACom) (pp. 1310-1315). Ieee.

49. Steele, B., Chandler, J., \& Reddy, S. (2016). Algorithms for data science (pp. 1-430). Springer.

50. Voulgaris, Z. (2014). Data scientist: the definitive guide to becoming a data scientist. Technics Publications.

51. Müller, A. C., \& Guido, S. (2016). Introduction to machine learning with Python: a guide for data scientists. " O’Reilly Media, Inc.”.

52. Cock, P. J., Antao, T., Chang, J. T., Chapman, B. A., Cox, C. J., Dalke, A., ... \& De Hoon, M. J. (2009). Biopython: freely available Python tools for computational molecular biology and bioinformatics. Bioinformatics, 25(11), 1422-1423.

53. Raschka, S. (2017). BioPandas: Working with molecular structures in pandas DataFrames. Journal of Open Source Software, 2(14), 279.

54. Rocklin, M. (2015, July). Dask: Parallel computation with blocked algorithms and task scheduling. In Proceedings of the $14^{\text {th }}$ python in science conference (No. 130-136).

55. Zheng, A., \& Casari, A. (2018). Feature engineering for machine learning: principles and techniques for data scientists. "O'Reilly Media, Inc.”.

56. Hackeling, G. (2017). Mastering Machine Learning with scikit-learn. Packt Publishing Ltd.

57. Géron, A. (2019). Hands-on machine learning with Scikit-Learn, Keras, and TensorFlow: Concepts, tools, and techniques to build intelligent systems. O'Reilly Media.

58. Nelli, F. (2018). Machine Learning with scikit-learn. In Python Data Analytics (pp. 313347). Apress, Berkeley, CA.

59. Purkait, N. (2019). Hands-On Neural Networks with Keras: Design and create neural networks using deep learning and artificial intelligence principles. Packt Publishing Ltd.

60. Menshawy, A. (2018). Deep Learning By Example: A hands-on guide to implementing advanced machine learning algorithms and neural networks. Packt Publishing Ltd.

61. Drabas, T., \& Lee, D. (2017). Learning PySpark. Packt Publishing Ltd.

62. Min, S., Lee, B., \& Yoon, S. (2017). Deep learning in bioinformatics. Briefings in bioinformatics, 18(5), 851-869.

63. Snyder, J. C., Rupp, M., Hansen, K., Müller, K. R., \& Burke, K. (2012). Finding density functionals with machine learning. Physical review letters, 108(25), 253002.

64. Brockherde, F., Vogt, L., Li, L., Tuckerman, M. E., Burke, K., \& Müller, K. R. (2017). Bypassing the Kohn-Sham equations with machine learning. Nature communications, $8(1)$, $1-10$. 
65. Li, L., Snyder, J. C., Pelaschier, I. M., Huang, J., Niranjan, U. N., Duncan, P., ... \& Burke, K. (2016). Understanding machine-learned density functionals. International Journal of Quantum Chemistry, 116(11), 819-833.

66. Dick, S., \& Fernandez-Serra, M. (2020). Machine learning accurate exchange and correlation functionals of the electronic density. Nature communications, 11(1), 1-10.

67. Mansimov, E., Mahmood, O., Kang, S., \& Cho, K. (2019). Molecular geometry prediction using a deep generative graph neural network. Scientific reports, 9(1), 1-13.

68. Ghosh, K., Stuke, A., Todorović, M., Jørgensen, P. B., Schmidt, M. N., Vehtari, A., \& Rinke, P. (2019). Deep learning spectroscopy: Neural networks for molecular excitation spectra. Advanced science, 6(9), 1801367.

69. Gao, P., Zhang, J., Peng, Q., Zhang, J., \& Glezakou, V. A. (2020). General Protocol for the Accurate Prediction of Molecular 13C/1H NMR Chemical Shifts via Machine Learning Augmented DFT. Journal of Chemical Information and Modeling, 60(8), 3746-3754.

70. Korolev, V., Mitrofanov, A., Korotcov, A., \& Tkachenko, V. (2019). Graph Convolutional Neural Networks as "General-Purpose" Property Predictors: The Universality and Limits of Applicability. Journal of Chemical Information and Modeling, 60(1), 22-28.

71. Wang, M., Wang, T., Cai, P., \& Chen, X. (2019). Nanomaterials discovery and design through machine learning. Small Methods, 3(5), 1900025.

72. Cole, J. M. (2020). A Design-to-device pipeline for data-driven materials discovery. Accounts of Chemical Research, 53(3), 599-610.

73. Choudhary, K., Bercx, M., Jiang, J., Pachter, R., Lamoen, D., \& Tavazza, F. (2019). Accelerated discovery of efficient solar cell materials using quantum and machine-learning methods. Chemistry of Materials, 31(15), 5900-5908.

74. Masood, H., Toe, C. Y., Teoh, W. Y., Sethu, V., \& Amal, R. (2019). Machine Learning for Accelerated Discovery of Solar Photocatalysts. ACS Catalysis, 9(12), 11774-11787.

75. Choudhary, K., Garrity, K. F., \& Tavazza, F. (2020). Data-driven discovery of 3D and 2D thermoelectric materials. Journal of Physics: Condensed Matter, 32(47), 475501.

76. Putin, E., Asadulaev, A., Ivanenkov, Y., Aladinskiy, V., Sanchez-Lengeling, B., AspuruGuzik, A., \& Zhavoronkov, A. (2018). Reinforced adversarial neural computer for de novo molecular design. Journal of chemical information and modeling, 58(6), 1194-1204.

77. Blaschke, T., Olivecrona, M., Engkvist, O., Bajorath, J., \& Chen, H. (2018). Application of generative autoencoder in de novo molecular design. Molecular informatics, 37(1-2), 1700123. 
78. Putin, E., Asadulaev, A., Ivanenkov, Y., Aladinskiy, V., Sanchez-Lengeling, B., AspuruGuzik, A., \& Zhavoronkov, A. (2018). Reinforced adversarial neural computer for de novo molecular design. Journal of chemical information and modeling, 58(6), 1194-1204.

79. Méndez-Lucio, O., Baillif, B., Clevert, D. A., Rouquié, D., \& Wichard, J. (2020). De novo generation of hit-like molecules from gene expression signatures using artificial intelligence. Nature communications, 11(1), 1-10.

80. Mercado, R., Rastemo, T., Lindelöf, E., Klambauer, G., Engkvist, O., Chen, H., \& Bjerrum, E. J. (2020). Graph Networks for Molecular Design. ChemRxiv preprint doi, 10.

81. Botu, V., Batra, R., Chapman, J., \& Ramprasad, R. (2017). Machine learning force fields: construction, validation, and outlook. The Journal of Physical Chemistry C, 121(1), 511522.

82. Noé, F., De Fabritiis, G., \& Clementi, C. (2020). Machine learning for protein folding and dynamics. Current Opinion in Structural Biology, 60, 77-84.

83. Gkeka, P., Stoltz, G., Farimani, A. B., Belkacemi, Z., Ceriotti, M., Chodera, J., ... \& Peter, C. (2020). Machine learning force fields and coarse-grained variables in molecular dynamics: application to materials and biological systems. arXiv preprint arXiv:2004.06950.

84. Sun, T., Zhou, B., Lai, L., \& Pei, J. (2017). Sequence-based prediction of protein protein interaction using a deep-learning algorithm. BMC bioinformatics, 18(1), 1-8.

85. Zeng, M., Zhang, F., Wu, F. X., Li, Y., Wang, J., \& Li, M. (2020). Protein-protein interaction site prediction through combining local and global features with deep neural networks. Bioinformatics, 36(4), 1114-1120.

86. Wang, Y. B., You, Z. H., Yang, S., Yi, H. C., Chen, Z. H., \& Zheng, K. (2020). A deep learning-based method for drug-target interaction prediction based on long short-term memory neural network. BMC Medical Informatics and Decision Making, 20(2), 1-9.

87. Wen, M., Zhang, Z., Niu, S., Sha, H., Yang, R., Yun, Y., \& Lu, H. (2017). Deep-learningbased drug-target interaction prediction. Journal of proteome research, 16(4), 1401-1409.

88. Geoffrey AS, B., Valluri, P. P., Sanker, A., Madaj, R., Davidd, H. A., Malgija, B., ... \& Mittal, B. (2020). Compound2Drug-a machine/deep learning tool for predicting the bioactivity of PubChem compounds.

89. Stepniewska-Dziubinska, M. M., Zielenkiewicz, P., \& Siedlecki, P. (2018). Development and evaluation of a deep learning model for protein-ligand binding affinity prediction. Bioinformatics, 34(21), 3666-3674.

90. Zhang, H., Liao, L., Saravanan, K. M., Yin, P., \& Wei, Y. (2019). DeepBindRG: a deep learning based method for estimating effective protein-ligand affinity. PeerJ, 7, e7362. 
91. Öztürk, H., Özgür, A., \& Ozkirimli, E. (2018). DeepDTA: deep drug-target binding affinity prediction. Bioinformatics, 34(17), i821-i829.

92. Ballester, P. J., \& Mitchell, J. B. (2010). A machine learning approach to predicting proteinligand binding affinity with applications to molecular docking. Bioinformatics, 26(9), 11691175 .

93. Cui, Y., Dong, Q., Hong, D., \& Wang, X. (2019). Predicting protein-ligand binding residues with deep convolutional neural networks. BMC bioinformatics, 20(1), 93.

94. Xie, R., Wen, J., Quitadamo, A., Cheng, J., \& Shi, X. (2017). A deep auto-encoder model for gene expression prediction. BMC genomics, 18(9), 845.

95. Guo, Y., Shang, X., \& Li, Z. (2019). Identification of cancer subtypes by integrating multiple types of transcriptomics data with deep learning in breast cancer. Neurocomputing, 324, 20-30.

96. Zhu, W., Xie, L., Han, J., \& Guo, X. (2020). The Application of Deep Learning in Cancer Prognosis Prediction. Cancers, 12(3), 603.

97. Zhou, J., Theesfeld, C. L., Yao, K., Chen, K. M., Wong, A. K., \& Troyanskaya, O. G. (2018). Deep learning sequence-based ab initio prediction of variant effects on expression and disease risk. Nature genetics, 50(8), 1171-1179.

98. Zhang, Z., \& Liu, Z. P. (2019, August). Identifying Cancer Biomarkers from HighThroughput RNA Sequencing Data by Machine Learning. In International Conference on Intelligent Computing (pp. 517-528). Springer, Cham.

99. van IJzendoorn, D. G., Szuhai, K., Briaire-de Bruijn, I. H., Kostine, M., Kuijjer, M. L., \& Bovée, J. V. (2019). Machine learning analysis of gene expression data reveals novel diagnostic and prognostic biomarkers and identifies therapeutic targets for soft tissue sarcomas. PLoS computational biology, 15(2), e1006826.

100. Mahapatro, G., Mishra, D., Shaw, K., Mishra, S., \& Jena, T. (2012). Phylogenetic tree construction for DNA sequences using clustering methods. Procedia engineering, 38, 13621366.

101. Fioravanti, D., Giarratano, Y., Maggio, V., Agostinelli, C., Chierici, M., Jurman, G., \& Furlanello, C. (2018). Phylogenetic convolutional neural networks in metagenomics. BMC bioinformatics, 19(2), 49.

102. Fiannaca, A., La Paglia, L., La Rosa, M., Renda, G., Rizzo, R., Gaglio, S., \& Urso, A. (2018). Deep learning models for bacteria taxonomic classification of metagenomic data. BMC bioinformatics, 19(7), 198.

103. Torrisi, M., Pollastri, G., \& Le, Q. (2020). Deep learning methods in protein structure prediction. Computational and Structural Biotechnology Journal. 
104. Kulmanov, M., \& Hoehndorf, R. (2020). DeepGOPlus: improved protein function prediction from sequence. Bioinformatics, 36(2), 422-429. 